\title{
Network growth models and genetic regulatory networks
}

\author{
D. V. Foster, ${ }^{1}$ S. A. Kauffman, ${ }^{2}$ and J. E. S. Socolar ${ }^{1}$ \\ ${ }^{1}$ Physics Department and Center for Nonlinear and Complex Systems, Duke University, Durham, NC, 27514 \\ ${ }^{2}$ Institute for Biocomplexity and Informatics, University of Calgary, Calgary, AB, Canada
}

(Dated: October 22, 2018)

\begin{abstract}
We study a class of growth algorithms for directed graphs that are candidate models for the evolution of genetic regulatory networks. The algorithms involve partial duplication of nodes and their links, together with innovation of new links, allowing for the possibility that input and output links from a newly created node may have different probabilities of survival. We find some counterintuitive trends as parameters are varied, including the broadening of indegree distribution when the probability for retaining input links is decreased. We also find that both the scaling of transcription factors with genome size and the measured degree distributions for genes in yeast can be reproduced by the growth algorithm if and only if a special seed is used to initiate the process.
\end{abstract}

\section{INTRODUCTION}

The manufacturing by cells of the proteins necessary for sustaining life is accomplished with the aid of molecular machinery that translates DNA nucleotide sequences, or genes, into their specified proteins at appropriate times. The first step in making a protein is the transcription process, in which the needed piece of RNA is formed from the relevant gene. The rate of transcription of a given gene can often be enhanced or suppressed by the presence of proteins that bind to the DNA near the gene, the gene's promoter region. Once transcription has taken place, the rate at which a given protein is produced may be further affected by the presence or absence of other proteins in the cell or other factors in the chemical environment. One way to approach the study of this complicated system of interacting molecules is to think of each gene, together with its promoter region, as an agent that interacts with other genes via protein-mediated interactions.

The logical structure of systems of many agents that exert causal influences on each other may be represented as a graph in which nodes represent agents and directed edges indicate the presence of a causal influence of one agent on another. In modeling the logic of the cell, for example, we may think of each node as representing a gene and each directed link as indicating that the concentration of the protein produced by one gene has some effect on the production rate of the other gene's protein. More precisely, a node represents a gene together with its promoter region. An incoming link indicates that under some circumstances a given protein can affect the transcription rate of a gene. The full set of genes (with promoter regions) and interactions forms a graph that may be called the genetic regulatory network. The dynam$i c s$ of the network is determined by parameters (reaction rates) associated with the links and by a function for each gene that determines the rate of protein production as a function of the concentrations of its regulators.

Genetic regulatory networks have, at least along some branches of evolution, grown over time from a relatively small ancestral genome to a vastly complex network of over 20,000 genes. We would like to know which features of real genetic regulatory networks are reflections of simple physical or mathematical laws as opposed to finely tuned solutions of specific problems faced during the evolutionary process. As a first step, we study a class of simple models of the growth process to see which network features arise purely from probabilistic effects when selection plays a minimal role. After discussing the general trends associated with the variation of certain parameters in the model, we compare the structures generated by the model to biological data and highlight some problematic issues in the interpretation of the data.

There is substantial evidence for the hypothesis that network growth occurs primarily via the duplication of genes and subsequent mutations of one or both members of the duplicate pair. [1] Under such mutations (or imperfect copying) the input and output links to a gene may not all be preserved as both the promoter region and the protein produced are altered. In addition, there is the possibility that a mutation in a gene will cause its protein to bind to a new promoter region or protein complex and thereby form a link that could not have been inherited during duplication alone. Similarly, a new link could be formed due to the mutation of a promoter region to a configuration that now binds a new protein. We refer to links that are generated by mutation as "innovated" links; those created via duplication are called "inherited." Studies of yeast and E. coli genomes suggest that innovation may account for as much as $50 \%$ of the links in the regulatory network. 2]

In modeling the growth of a genetic regulatory network one must incorporate some assumptions about the effect of natural selection. We make several simplifying assumptions concerning separations of time scales. As we are interested here in the evolution of the network structure that occurs over many generations, we ignore the time scale corresponding to cellular processes and the lifetime of individual organisms. Our evolutionary model assumes three additional scales. First, there is the typical time required for a duplicated gene to drift via mutation to a new stable gene. Call this the "mutation" time scale. Second, we assume a much longer time scale 
required for the occurrence of duplication events. That is, we assume that after a duplication event whatever mutation is going to occur in the duplicated gene happens before any other duplication event occurs. Finally, once a gene has mutated a certain amount and thereby found a niche for itself in the cell, natural selection is assumed to keep it stable over time scales long compared to the duplication time scale. Though this is somewhat of a caricature of evolutionary processes at the genetic level, it has the virtue of conceptual clarity. We note that this model applies only to duplication events that ultimately lead to an increase in the size of the genome. It does not attempt to model duplications that lead to adaptive radiation and eventual selection of a single duplicated gene as the fittest [3].

Consistent with the above assumptions, our networks grow via duplication/mutation events. When a gene $G$ (together with its promoter region) is duplicated to create $G^{\prime}$, it is assumed that $G$ remains fixed while $G^{\prime}$ mutates (or that a portion of it is not copied faithfully) so that some of the duplicated input and output links at $G^{\prime}$ cease to function. The parameters in our growth model are the probabilities of retaining inherited links after mutation and the probabilities of innovating links. Note that the binding of a protein to a promoter region of DNA breaks the symmetry between input and output inheritance. In the output case, the issue is whether a mutation causes changes in a protein that significantly decrease its binding affinity to unchanged portions of DNA (or perhaps to other proteins). In the input case, the issue is whether the mutation in the promoter region decreases the binding affinity of a protein that has not changed.

We consider three classes of models: (1) partial duplication, in which only a subset of links is inherited during a growth event; (2) partial duplication with constant probability innovation, in which innovation probabilities are independent of the characteristics of the candidate nodes to be linked; and (3) partial duplication with "richgets-richer" innovation, in which nodes with more inputs have higher probabilities of forming innovative input links. The set of models we study includes as special cases several models studied previously. [4, 5, 6, 6, 8, 8, 10, 11] In the present work we emphasize the importance of different probabilities for input and output inheritance and focus on features of finite networks rather than scaling laws for arbitrarily large networks.

For each case we study numerically the statistical features of networks of up to 2000 nodes grown from seeds with 10 nodes or, for reasons that will become clear later, 100 nodes. In several cases we provide theoretical calculations supporting the numerical results. We find that certain choices of parameters result in networks that have input and output degree distributions similar to those reported for yeast cells and simultaneously match results on the scaling of the numbers of transcription factors with genome size. Our results suggest that a simple process that totally neglects any specific information about the biological function of individual genes can produce a realistic network, but only if the process starts from an appropriate seed.

Before turning to the details of the growth algorithm, we wish to emphasize the importance of considering the precise meaning of a link in the network, since this has direct implications for the comparison to experimental data. A link between genes could be taken to mean that the protein product of a gene is a transcription factor that binds directly to a gene's promoter region. This interpretation allows the network to be determined with relatively straightforward experiments that test for the binding of a given protein to a given promoter region. (See, for example, [12, 13].) It is also possible, however, for proteins that fail to bind directly to DNA to assert regulatory control through the formation of protein complexes at a promoter region or even through participation in chemical processes that occur far from the DNA. For purposes of simulating genome-wide transcriptional dynamics, all causal relationships between the expression levels of two genes should be represented as links in the network. The difference between model parameters germane to these two interpretations is discussed in detail below.

\section{GROWTH OF DIRECTED NETWORKS THROUGH PARTIAL DUPLICATION}

We make the following definitions:

a family of nodes is the set of all nodes arising through a chain of duplications of a single ancestor in the seed;

a constitutive node is a regulator that has no inputs.

a regulator is any node that has at least one output;

an inert node is a the result of a duplication event in which all inputs and outputs are deleted (a constitutive non-regulator);

a transcription factor is a regulator that has at least one output that has been inherited, perhaps through several generations, from a seed regulator that has been designated a transcription factor.

In biological terms, a constitutive node represents a gene whose expression level never changes or else changes only in response to external environmental variables. An inert node may represent either a nonfunctional bit of (junk) DNA or a gene that responds to environmental factors but remains completely independent of the activity of any other genes. A transcription factor represents a gene whose protein binds to DNA, and it is assumed that mutations never create new transcription factors from other types of regulators.

The partial duplication model is implemented according to the following procedure. We define a time step to be the time between duplication events. At each time 
step a gene $G$ is chosen at random from the network and duplicated, forming a gene $G^{\prime}$ that has all the same input and output links as $G$. One of these identical nodes, say $G^{\prime}$, is then assumed to mutate. Each input link inherited from $G$ is independently tested and kept with probability $c_{i}$, and each output link with probability $c_{o}$. If $G^{\prime}$ should lose all of its inputs and outputs, it is considered to have lost all its function, and thus is removed from the network entirely. As mentioned above, there is no physical symmetry requiring $c_{i}=c_{o}$.

We can gain some intuition about the effects of the parameters by studying some limiting cases that permit analytical solutions. The simplest of these is $c_{i}=c_{o}=1$, the case in which all links are kept after every duplica- tion event. In this case all nodes in the same family have exactly the same set of inputs and outputs. The degree distributions will consist of delta functions whose positions depend on the seed network chosen and the number of duplication events that have occurred within each family.

For $c_{i}$ and $c_{o}$ less than unity, a master equation describes the evolution of the degree distributions during growth. 8] Let $t$ represent the total number of nodes in the network. Advancing $t$ by one corresponds to a single duplication event. Let $N_{i}(t, k)$ and $N_{o}(t, k)$ represent the number of nodes at "time" $t$ having $k$ inputs and outputs, respectively. On average, we have

$$
\begin{aligned}
& N_{i}(t+1, k)=N_{i}(t, k)+\frac{1}{t} \sum_{k^{\prime}=k}^{t}\left(\begin{array}{c}
k^{\prime} \\
k
\end{array}\right) c_{i}^{k}\left(1-c_{i}\right)^{k^{\prime}} N_{i}\left(t, k^{\prime}\right)+\frac{c_{o}}{t}\left[(k-1) N_{i}(t, k-1)-k N_{i}(t, k)\right] . \\
& N_{0}(t+1, k)=N_{o}(t, k)+\frac{1}{t} \sum_{k^{\prime}=k}^{t}\left(\begin{array}{c}
k^{\prime} \\
k
\end{array}\right) c_{o}^{k}\left(1-c_{o}\right)^{k^{\prime}} N_{o}\left(t, k^{\prime}\right)+\frac{c_{i}}{t}\left[(k-1) N_{o}(t, k-1)-k N_{o}(t, k)\right] .
\end{aligned}
$$

The sum on the right hand side of the first equation represents probability of the addition of a node with $k$ inputs due to the duplication and subsequent mutation of a node with $k^{\prime}$ inputs. The $c_{o}(k-1) N_{i}(t, k-1) / t$ term is the probability that the duplicated node is one of the $(k-1)$ inputs to a node $K$ and the inherited output is kept, so that the number of inputs to $K$ is incremented to $k$. The last term comes from the possibility of adding a new input to a node that already has $k$ inputs.

In the limit of large $t$, the sums in (11) can be approximated by

$$
\sum_{k^{\prime}=k}^{t}\left(\begin{array}{l}
k^{\prime} \\
k
\end{array}\right) c^{k}(1-c)^{k^{\prime}} N\left(t, k^{\prime}\right)=\frac{1}{c} N(t, k / c),
$$

where we have assumed that $N\left(t, k^{\prime}\right)$ does not vary significantly over the range of $k^{\prime}$ values that have an appreciable probability to yield $k$ inputs after mutation [4]. The factor of $1 / c$ comes from the fact that there are $1 / c$ values of $k^{\prime}$ for which $\left\lfloor c k^{\prime}\right\rfloor=k$. This approximation allows for rapid iteration of the master equation, enabling numerical studies of distributions for large $t$. (For analytical results on master equations of this type, see $[8]$.)

For $c_{i}=c_{o} \equiv c$ the two master equations become identical and the asymptotic forms of the input and output distributions are the same. For $0.2 \lesssim c \lesssim 0.7$, the approximate master equation gives an accurate estimate of $N(t, k)$ for network sizes $t>100$, as shown in Figure 1 For $c$ outside this range, however, the degree distribution predicted by iterating the approximate equation only matches simulation data for large values of $k$, as expected.
As $c$ approaches zero, the duplication/mutation process becomes essentially equivalent to the preferential attachment growth algorithm [6] that is known to produce networks with scale free degree distributions. The only difference is that the duplication process produces a large number of inert nodes, whereas preferential attachment counts only those nodes that do get linked to the existing network. For sufficiently small $c$, the probability of producing a node with more than one input or output via partial duplication is negligible (on the order of $c^{2}$ ), so each new node (that is not inert) is added to the network with a single input or output. The probability of forming a new input to node $G$ is proportional to the probability of selecting an input node to $G$ for duplication, which in turn is proportional to the number of inputs that $G$ already has. For $c \rightarrow 0$, ignoring the production of linkless (inert)nodes leads to:

$$
N(t+1, k)=N(t, k)+\delta(k-1)+\frac{1}{t}[(k-1) N(t, k-1)-k N(t, k)] .
$$

The total number of links in the connected part of the network approaches $t$ as $c$ gets small, so this master equation is equivalent to the one given for preferential attachment by Barabasi et al., which is known to yield a scale-free distribution $N(t, k)=k^{-\gamma}$ with $\gamma=3$. [5, []

For $c=0.5$, our model is equivalent to one proposed by Dorogovtsev et al, who predicted scale free behavior in the degree distribution function, with the frequency of occurrence of indegree (or outdegree) $k$ decaying like $k^{\sqrt{2}}$. [4] This scale free behavior should occur, however, only over the domain of very large $t$ and $k$. The systems we 


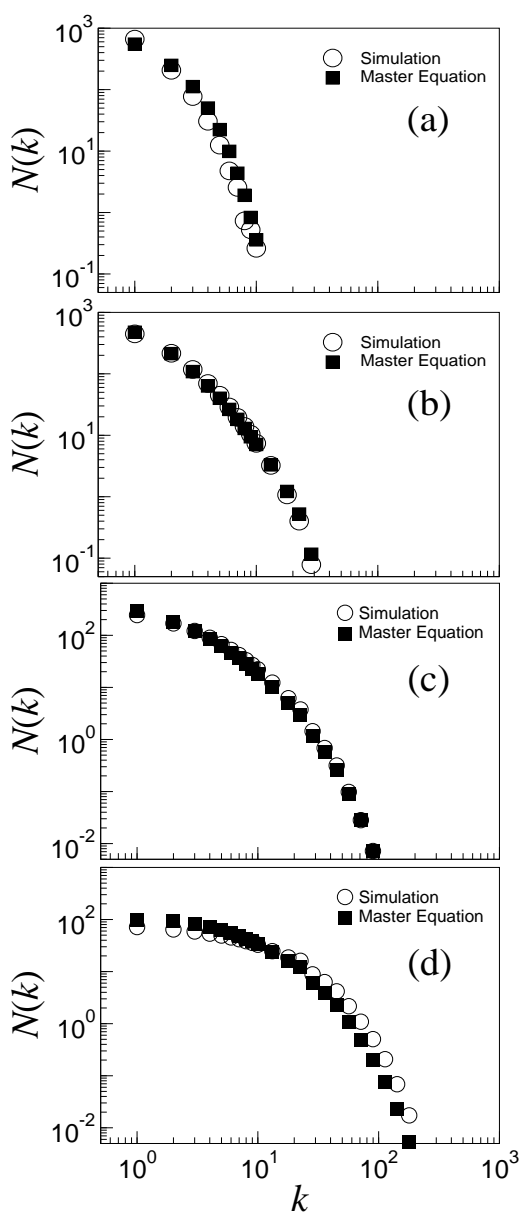

FIG. 1: Simulated (circles) and predicted (squares) degree distribution functions for different values of $c$. (a) $c=0.1$; (b) $c=0.3$; (c) $c=0.5$; and (d) $c=0.7$. For models with no innovation and $c_{i}=c_{o}$ the indegree and outdegree distributions are identical.

have studied are not large enough to show the predicted scaling, as we can see from the (c) panel in Figure 1 References [9, 10, 11] also provide analytical results on the asymptotic scaling properties for small $c$ and failure of self-averaging for large $c$ in similar models.

\section{A. Effects of different values for $c_{i}$ and $c_{o}$}

Holding $c_{o}$ constant at 0.5 , it is interesting to see what happens as $c_{i}$ is varied. To compare the distributions for different parameter values we choose to keep the total number of (non-inert) nodes fixed at 1000. That is, we simulate the network growth (not the approximate master equations) from a seed of 10 nodes with 10 randomly assigned connections, discarding inert nodes and stopping when the network contains 1000 nodes. The data shown in Fig. 2] are averages taken over 100 networks.

Over the range of sizes probed, the indegree distributions are roughly scale-free for smaller values of $c_{i}$ as seen

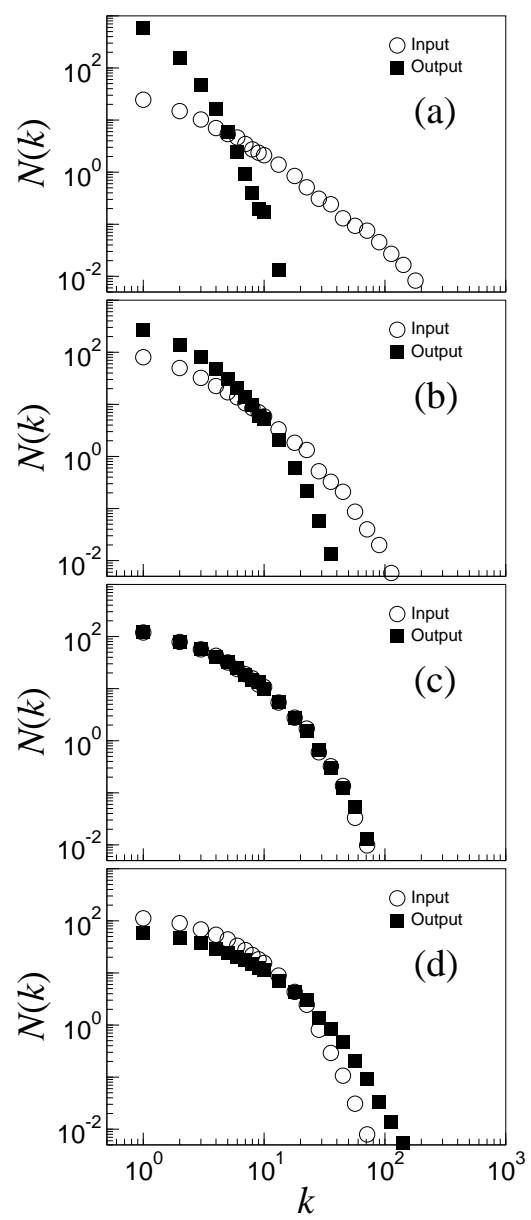

FIG. 2: Indegree (circles) and outdegree (squares) distribution functions for $c_{o}=0.5$ and different values of $c_{i}$ : (a) $c_{i}=0.1 ;$ (b) $c_{i}=0.3$; (c) $c_{i}=0.5$; and (d) $c_{i}=0.7$. Each data point represents the average number of nodes with a given number of inputs in an ensemble of 100 networks.

in Fig. 2(a). We note that the scaling exponent suggested by these data is approximately $\gamma=1$, but the systems are not large enough to be described by the asymptotic scaling regime of the relevant master equation, so we do not expect this scaling to persist to substantially larger system sizes. A surprising result is that decreasing $c_{i}$ actually tends to broaden the tail of the indegree distribution. One may have expected a shift of the distribution favoring smaller indegrees because the probability of keeping a large number of inputs in a given duplication event decreases with decreasing $c_{i}$. There is a competing effect, however, associated with the increased production of inert nodes, which are not counted as part of the 1000 nodes that constitute the final network. Because nodes with small indegrees tend to produce inert nodes, the probability of a node being duplicated is skewed toward nodes with high indegrees. In the limit of very small $c_{i}$, the situation is similar to the preferential attachment limit discussed above, where nodes with more inputs are more likely to receive new inputs when another node is 
duplicated. The difference here is that the newly created node may have many outputs rather than the fixed number stipulated in standard preferential attachment models.

Note that Fig. 2(c) is not identical to Fig. 1(c), even though they correspond to the same parameter values. This is because of the difference in the criteria used for determining the sizes of the networks in the ensemble. For purposes of comparing distributions to those predicted by the master equation, we must include inert nodes in the simulation. That is, inert nodes are counted as contributing to the system size. For the purpose of examining an ensemble of networks with a given number of genes, however, we do not count the inert nodes. Interpreted in this context, the data shown in Fig. 目(c) correspond to an ensemble with a distribution of networks sizes, all smaller than 1000 .

\section{EFFECTS OF INNOVATION}

The pure partial duplication model does not contain a mechanism for innovation of new links. We now consider two models for representing the general effects of innovation. The first model is similar to that one introduced by Solé [7]: each time a new node is created, every possible input and output link it might form is tested and kept with independent probability $p$. In the second model, the probability of keeping a tested link is assumed to depend on the indegree of the node at the input side of the link; nodes with more inputs are assumed to have a higher probability of accepting new inputs. The motivation for the latter comes from the ability of proteins to form complexes or interact in ways that affect transcription, which suggests that if more proteins participate in the regulation of a given gene, there are more opportunities for a new protein to exert a regulatory influence. Note that this is not true on the output side; the probability that a given protein will participate in a particular regulatory link should not change just because that protein begins to participate in additional links.

\section{A. Constant probability innovation}

Innovation is added to the partial duplication model as follows. At each time step a gene $G^{\prime}$ is produced by partial duplication. $G^{\prime}$ is then given a chance to develop an innovative input from all of the transcription factors in the network. Note that in this model all regulators are transcription factors. It is impossible for a node with no outputs to become a regulator. If gene $G^{\prime}$ has inherited outputs (and thus is a transcription factor), then it is given a chance $p$ to bind to every node in the network. If $G^{\prime}$ has no inputs or outputs after all inherited and innovated links are tested, it becomes inert and is assumed to remain inert forever; it can no longer receive innovated inputs.

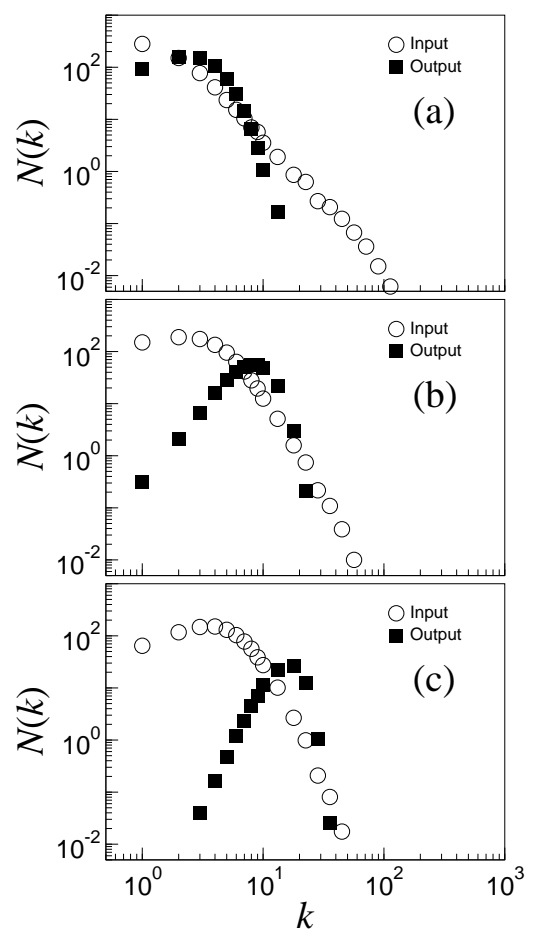

FIG. 3: Indegree (circles) and outdegree (squares) distribution functions for $c_{o}=0.5$ and $c_{i}=0.1$, with various values of $p$ : (a) $p=.001$; (b) $p=.005$; (c) $p=.01$. Each different value of $p$ results in a different percentage of innovated links: (a) $39.1 \%$ (b) $59.8 \%$ (c) $64.6 \%$.

For the present study, networks were grown until the number of non-inert nodes reached $N=1000$. Figs. 3 and 4 show the indegree and outdegree distributions for $c_{o}=0.5$ and $c_{i}=0.1$ and 0.5 , respectively, for several values of $p$.

Comparing Figs. 3 and 4 to Fig. 2 we see that innovation serves to shift the weight in the outdegree distribution to larger values of $k$ and produces a peak at nonzero $k$. This qualitatively matches the yeast data of Lee et al., who find that the output distribution has a peak at a value near 30. 12. However, innovation also reduces the weight in the tail of the indegree distribution. This somewhat surprising result is due to the fact that innovation decreases the number of nodes with only a single input, thereby decreasing the rate at which duplications yield inert nodes, which in turn decreases the number of duplications of high indegree nodes.

\section{B. Rich-gets-richer innovation}

As mentioned above, links corresponding to the direct bindings of transcription factors to DNA do not exhaust the possible sources of regulatory control. To model the networks observed in experiments that detect the influence of protein interactions and other regulatory effects, one must consider models in which non-regulatory nodes 


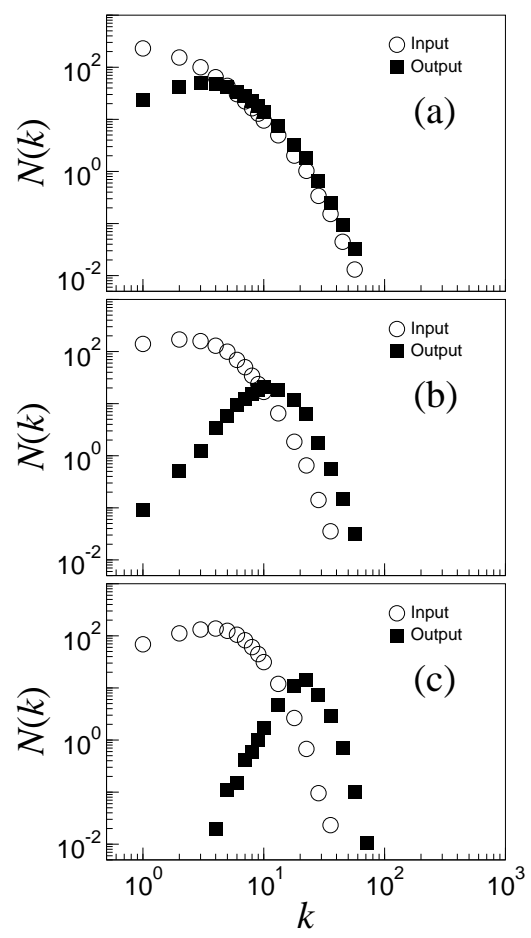

FIG. 4: Indegree (circles) and outdegree (squares) distribution functions for $c_{o}=0.5$ and $c_{i}=0.5$, with various values of $p$ : (a) $p=.001$; (b) $p=.005$; (c) $p=.01$. Each different value of $p$ results in a different percentage of innovated links: (a) $16.2 \%$ (b) $36.5 \%$ (c) $43.2 \%$.

can develop outputs via innovation. If protein interactions are an important source of regulatory control, we might expect that any gene could possibly innovate an output to any other gene, and that genes with more inputs would be more likely to gain further inputs. This "rich-gets-richer" effect may produce scale free indegree distributions, as in simpler preferential attachment models.

One might imagine many different ways in which the probability of receiving an innovated input could depend on the indegree of a node. The situation of interest, though, is one in which the interactions between nodes are determined by physical properties of molecules and therefore should not depend on the order in which nodes were added to the system. That is, the probability that a link from $G$ to $H$ is innovated may depend on the number of inputs to $H$, but should not depend on whether those inputs were generated before or after $G$ was created. This constraint restricts the class of models considerably, as described below.

The model is implemented according to the following procedure. At each time step, first a gene $G$ is chosen at random from the network and partial duplication occurs with probabilities $c_{i}$ and $c_{o}$ as above. Each gene in the network is then tested to see if it innovates an input to $G^{\prime}$ and/or receives an innovative input from $G^{\prime}$. Whenever a new gene $G^{\prime}$ is created, it is given a probability $p(n)$ of having an output to each gene $H$, where $n$ is the number of inputs $H$ already has. (Note that $p(0)$ does not vanish. There is some probability that the new gene will regulate a gene that previously had no inputs.) In addition, any time that any gene $J$ acquires one or more new inputs, whether via duplication or innovation, all other genes $K$ in the network are given probability $q(\Delta)$ of forming a new input link to $J$, where $\Delta$ is the number of inputs that $J$ has gained since the last time the formation of an innovative link from $K$ to $J$ was tested. This latter process is iterated until a complete pass through the network generates no new links. Multiple time steps are performed until the network contains $N$ genes with at least one input or output link.

To ensure that the probabilities of links being present are independent of the order in which new innovations are attempted, we must choose

$$
\begin{aligned}
& p(n)=1-e^{-(1+n) / n_{0}} \\
& q(\Delta)=1-e^{-\Delta / n_{0}}
\end{aligned}
$$

where $1 / n_{0}$ gives the probability that a gene with no inputs will obtain an input from any particular gene. The exponential form of $q(\Delta)$, means that $\left[1-q\left(\Delta_{1}\right)\right][1-$ $\left.q\left(\Delta_{2}\right)\right]=\left[1-q\left(\Delta_{1}+\Delta_{2}\right)\right]$ for any $\Delta_{1}$ and $\Delta_{2}$. This ensures that the probability of there being no link between two genes is independent of the number of times the potential link was tested during the growth process. Thus our model is a self-consistent growth algorithm in which the probability of adding a new link can always be determined without worrying about the order in which links are tested and added to the system.

The amount of innovation and the values of $c_{i}$ and $c_{o}$ are roughly similar between Fig. 3 (a) and Fig. 5 (a), Fig. (b) and Fig. 囵(c). These pairs of figures show that rich-gets-richer innovation broadens the indegree distribution compared to constant probability innovation, as expected when high indegree nodes are favored for innovated inputs. We also note the general trend that richgets-richer innovation gives rise to a less pronounced peak in the outdegree distribution and to clearer power law tails.

The differences between constant probability innovation and rich-gets-richer innovation may be relevant for the modeling of biological data. At the very least, these differences highlight the importance of obtaining a clear understanding of the types of regulatory interactions that are included in experimental reports on the structure of genetic regulatory networks.

\section{Selecting a starting seed}

The discussion and results above focused on generic behaviors expected when a network has grown to many times the size of the initial seed. Unfortunately, it appears that no choices of the parameters $c_{i}, c_{o}$, and $p$, can reproduce degree distributions qualitatively similar 


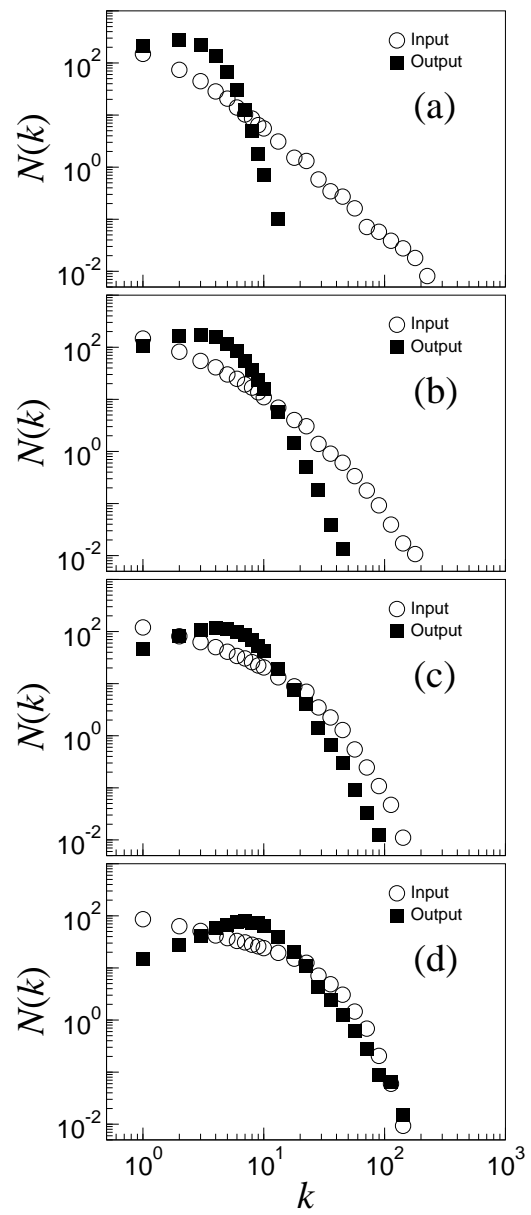

FIG. 5: Indegree (circles) and outdegree (squares) distribution functions for $c_{o}=0.5$ and different values of $c_{i}$ : (a) $c_{i}=0.1$; (b) $c_{i}=0.3$; (c) $c_{i}=0.5$; and (d) $c_{i}=0.7$, with $n_{0}=3000$ in the rich-gets-richer innovation model. The percentage of innovated links is (a) $48.3 \%$; (b) $41.4 \%$; (c) $37.5 \%$; (d) $35.3 \%$.

to those reported for yeast in experiments measuring transcription factor binding. In those experiments, it is found that the output distribution is extremely broad, including an appreciable number of nodes with more than 130 outputs, while the input distribution remains quite narrow, containing very few nodes with more than about 10 inputs. 12, 14]

There is, however, another piece of biological evidence that suggests that real genetic regulatory networks cannot be in the asymptotic large $N$ regime. Nimwegen has observed that the number of transcription factors in an organism scales like $N^{\alpha}$, with alpha $\approx 1.26$ for eukaryotes and $\alpha \approx 1.87$ for bacteria, where $N$ is the size of the genome. 15] A scaling law of this type with $\alpha$ greater than unity is impossible for arbitrarily large genome sizes since the number of transcription factors cannot exceed the total number of genes. We are thus led to consider models in which the number of transcription factors is initially quite small compared to the total number of nodes

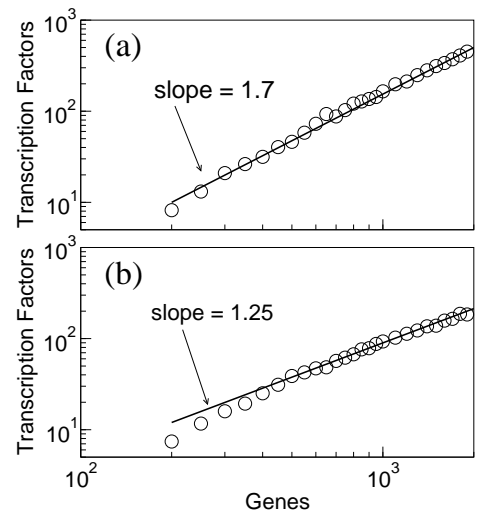

FIG. 6: Growth rate of the number of transcription factors for the parameters $c_{i}=0.2, c_{o}=0.5$, with (a) $p=0$ and (b) $p=0.005$. The networks are grown from a seed of one regulator linked to 99 other nodes. Solid lines show the scaling laws consistent with Nimwegen's analysis of (a) bacteria and (b) eukaryotes.

in the network.

We study networks of 2000 nodes grown from a seed consisting of a single node that regulates itself and 99 other non-regulating nodes. This is roughly consistent with an extrapolation from Nimwegen's observations to genomes with only 100 genes.

Since we are now considering data that identify transcription factors rather than all regulatory interactions, we work with the constant probability innovation model. As seen in Fig. [6 the number of transcription factors does indeed grow roughly as a power law, with an exponent that depends on the value of $c_{o}$ and $p$. The straight lines shown on the plots indicate scaling exponents roughly consistent with Nimwegen's reported values. (No attempt was made to search parameter space for optimal fits to Nimwegen's exponent for bacteria.)

In Fig. [6] one can see evidence for a region of quadratic scaling for very small system sizes, implying that when a gene is selected for duplication, the result is twice as likely to be kept in the genome if it is a transcription factor than if it is not. 15. We can understand this effect in the context of our model as follows. If $c_{o}$ is sufficiently large and transcription factors have many outputs, the chance that partial duplication of a transcription factor will result in an inert node is negligible. On the other hand, most genes have rather few inputs, so the probability of duplication of a non-transcription factor leading to an inert node is appreciable and is also sensitive to $c_{i}$. Since duplications of transcription factors increase the number of inputs to many of the nodes, exact calculation of the rate at which inert nodes are generated is difficult. We find numerically that $c_{i} \approx 0.2$ leads to an initial growth phase with roughly quadratic scaling.

It is interesting to compare the degree distributions obtained from the model with parameters that yield a scaling exponent consistent with Nimwegen's analysis of eukaryotes to the distributions reported for yeast. 12] 


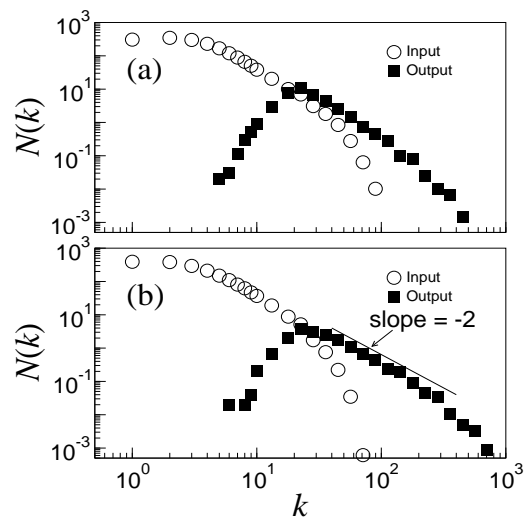

FIG. 7: (a) Indegree (circles) and outdegree (squares) distribution functions for the model parameters equal to those of Fig. 6lb): $c_{o}=0.5, c_{i}=0.2$ and $p=0.005$. (b) Same as (a) except $c_{i}=0.3$. The networks are grown from a seed of one regulator linked to itself and 99 other nodes.

Fig. [7 shows that we obtain an indegree distribution with no extended power-law tail together with an outdegree distribution with a broad power-law tail. The widths and shapes of the distributions are roughly consistent with those observed for yeast, including such features as the power-law outdegree tail with exponent near 2 and a slower-than-exponential decay at small indegree that is rapidly cut off above a maximum near 30 in the outdegree distribution. For the model parameters used here, approximately $28 \%$ of the links in the system are formed via innovation.

We note that Harbison et al. 14], using a different approach from Lee et al. 12], report data on outdegrees for 203 transcription factors that show a distribution extending to approximately 300 , but appearing to have an exponential form and no peak. In the Harbison data, however, outdegrees are determined as the union of results of different preparations that show wide variations in the outdegrees of individual genes. A third source of data on the yeast network is available from Milo et al. [13. Careful comparative analysis of all of the available data is beyond the scope of the present work.

\section{CONCLUSIONS}

We have introduced and studied two classes of growth algorithms for networks with directed links. Both involve partial duplication of nodes as the sole growth mechanism and separate parameters determining the probabilities of keeping inputs and outputs of the duplicated node. In one case, the opportunities for the innovation of new links are given a constant probability and every possible output link is tested exactly once during the growth. In the other, nodes with more input links are given a higher probability of receiving new inputs, and potential input links are re-tested every time a node receives a new input. In the context of our simplified description of genome growth, the first model is appropriate for studying transcription factor binding networks, where output links correspond only to direct binding of a protein to DNA. The second model corresponds to the full network of regulatory interactions, in which some proteins that do not bind to DNA can still exert regulatory control.

We have studied indegree and outdegree distributions and the relation between the number of transcription factors and system size in networks of up to 2000 nodes grown from random seeds with 10 nodes or special seeds with 100 nodes. Some counterintuitive results concerning the effects of various parameters on the degree distributions were observed and explained. Parameters were found that produce networks with degree distributions similar to those reported for yeast cells and plausibly realistic scaling laws for the number of transcription factors as a function of genome size in eukaryotes.

For our growth model, the production of realistic networks requires a seed in which many nodes are regulated by a single transcription factor with a self-input. While we do not know the detailed regulatory network structure of any ancestral organism with only 100 genes, it is plausible to suggest that early simple organisms required relatively few regulatory genes that controlled a large number of structural genes.

Several features of transcriptional network architecture would be natural candidates for further study. We have not yet analyzed the frequency of occurrence of small network motifs or clustering statistics. It is known that partial duplication induces strong local correlations in a model with identical probabilities for retaining input and output links. 7] We conjecture that minor modifications to our model favoring innovation between genes that share a neighbor could account for higher clustering coefficients and favor local motifs without altering the overall degree distributions or transcription factor scaling laws.

We have explored the behavior of a class of network growth models that may be relevant for understanding the structure of genetic regulatory networks. Our results indicate that several nontrivial features of presently available biological data can arise simply from probabilistic growth rules with no notion of optimization due to selection coming into play. This suggests that statistical features of real genetic regulatory networks may be determined by physical or biochemical parameters rather than careful tuning through natural selection.

This is not to say that natural selection plays no role in determining which networks survive and prosper. Among the ensemble of networks generated by our growth models, there are many possibilities for variation. For example, the causal influences indicated by links in our networks, and hence the dynamical properties of the network, may evolve under selection pressures via small mutations that do not affect the network architecture. Nevertheless, it is interesting to see that simple probabilistic growth rules can account for an ensemble of possibilities available to the fitness selection process. 16] 


\section{Acknowledgments}

S. K. thanks the Department of Cell Biology and Physiology at the University of New Mexico, Albuquerque, for support during the early stages of this work. This material is based upon work supported by the National Science Foundation under Grant No. PHY-0417372.
[1] B. Lewin, Genes VII (Oxford University Press, 2000).

[2] S. N. Teichmann and M. M. Babu, Nature Genetics 36, 496 (2004).

[3] M. P. Francino, Nature Genetics 37, 573 (2005).

[4] S. N. Dorogovtsev, J. F. F. Mendes, and A. N. Samukhin, Europhys. Lett. 57, 334 (2002).

[5] S. N. Dorogovtsev, J. F. F. Mendes, and A. N. Samukhin, Phys. Rev. Lett. 85, 4633 (2000).

[6] R. Albert and A.-L. Barabasi, Science 286, 509 (1999).

[7] R. P. Satorras, E. D. Smith, and R. V. Sole, J. Theor. Biol. 222, 199 (2003).

[8] P. L. Krapivsky, G. J. Rodgers, and S. Redner, Phys. Rev. Lett. 86, 5401 (2001).

[9] P. L. Krapivsky and S. Redner, Phys. Rev. E 71, 036118 (2005).

[10] I. Ispolatov, P. L. Krapivsky, and A. Yuryev, Phys. Rev. E 71, 061911 (2005).

[11] I. Ispolatov, P. L. Krapivsky, I. Mazo, and A. Yuryev,
New J. Phys. 7, 145 (2005).

[12] T. I. Lee, N. J. Rinaldi, F. Robert, D. T. Odom, Z. Bar-Joseph, G. K. Gerber, N. M. Hannett, C. R. Harbison, C. M. Thompson, I. Simon, et al., Science 298, 799 (2002), URL http://web.wi.mit.edu/young/regulator_network/

[13] R. Milo, S. Shen-Orr, S. Itzkowitz, N. Kashtan, D. Chklovskii, and U. Alon, Science 298, 824 (2002), URL http://www . weizmann . ac.il/mcb/UriAlon/

[14] C. T. Harbison, D. B. Gordon, T. I. Lee, N. Rinaldi, K. D. Macisaac, T. D. Danford, N. M. Hannett, J.-B. Tagne, D. B. Reynolds, J. Yoo, et al., Nature 431, 99 (2004), URL http://jura.wi.mit.edu/young_public/regulatory_code/ProtGen

[15] E. van Nimwegen, Trends in Genetics 19, 479 (2003).

[16] S. Kauffman, J. Theor. Biol. 230, 581 (2004). 